DOI: 10.17516/1997-1397-2020-13-2-141-150

УДК 517.9

\title{
Root Locus of Algebraic Equations
}

\section{Valeriy I. Bykov* \\ Svetlana B. Tsybenova ${ }^{\dagger}$}

Emanuel Institute of Biochemical Physics RAS

Moscow, Russian Federation

Received 28.02.2017, received in revised form 18.10.2018, accepted 15.01.2020

Abstract. The locus of real and complex roots of algebraic equations are constructed in this paper. Calculations of specific equations show that the location of their roots depends on the type of equation.

Keywords: algebraic equation, real root, complex root, root locus, nonlinear equation with parameters.

Citation: V.I.Bykov, S.B.Tsybenova, Root Locus of Algebraic Equations, J. Sib. Fed. Univ. Math. Phys., 2020, 13(2), 141-150. DOI: 10.17516/1997-1397-2020-13-2-141-150.

\section{Introduction}

In this paper, we consider some of specific algebraic equations. However, our experience shows that the reported examples allow us to understand the structure and features of the geometric location of the roots under changes of parameters in the general case.

Systems of nonlinear equations with parameters arise, for example, in the analysis of the stability of stationary equations of chemical kinetics or in mathematical models of chemical technologies [1-4]. From a formal point of view, parametric analysis of specific mathematical model leads to the study of the geometric location of roots of nonlinear (including algebraic) equations [5-11].

In this paper we consider specific, but very important, case of an algebraic equation of the form:

$$
F(z, p)=0
$$

where function $F$ is a polynomial, $z$ is unknown variable. $p$ is a parameter which is varied through a wide rage: $-\infty<p<+\infty$. As parameter $p$ changes $z(p)$ defined by equation (1) can continuously change (deformation) or change abruptly (reconstruction, bifurcation). Thom's classification theorem $[12,13]$ gives enumeration of possible local structures at bifurcation points. However, it is often important to know the quantitative characteristics of possible deformations of the solutions $z$ under changes of parameters $p$ in a wide interval. For example, it is important to determine not only the stability of steady states but also the degree of their stability and the stability margin of phase $[2,3]$.

\footnotetext{
*vibykov@mail.ru https://orcid.org/0000-0003-0775-385X

$\dagger$ https://orcid.org/0000-0001-5599-0580

(c) Siberian Federal University. All rights reserved
} 


\section{General analysis}

The main problem of the qualitative and numerical analysis of systems of nonlinear equations with parameter (1) is to construct the root locus $z(p)$. As a rule, the problem to construct the inverse relation

$$
p=p(z)
$$

is a more simple problem.

Construction of the root locus of algebraic equation

$$
a_{n} z^{n}+a_{n-1} z^{n-1}+\ldots+a_{1} z+a_{0}=0,
$$

is considered, where $a_{i}$ are real coefficients. Without loss of generality, instead of (3) the following algebraic equation

$$
z^{n}+a_{n-1} z^{n-1}+\ldots+a_{1} z+1=0 .
$$

is considered. Taking one of the coefficients in (4) as parameter $p$, rewrite this equation in the following way

$$
p=z^{n-k}+\ldots+\frac{1}{z^{k}}
$$

where $n<k<1$ and $z \in(-\infty,+\infty)$. For each fixed $k$, parameter $p$ is a function of real variable $z$. Relation (5) allows us to determine the inverse function

$$
z=\varphi(p) \text {. }
$$

Relation (5) allows us to relatively easy obtain $\varphi(p)$ which is the locus of real roots of original algebraic equation (4). The locus of complex roots $z$ of this equation under changes of parameter $p$ can be constructed from the condition

$$
\operatorname{Im} p(z)=0
$$

The simplest problem for equation (3) is to construct the locus of real and complex roots of algebraic equations with three monomials

$$
z^{n}-p z^{n-k}+1=0,
$$

where $1<k<n ; n$ and $k$ are integers. Let us note that quadratic and cubic equations without loss of generality quite fit in case (8). From equation (8) we have

$$
p=z^{k}+\frac{1}{z^{n-k}} .
$$

Relation $p(z)$ defined in (9) specifies the locus of real roots $z(p)$ or rather its inverse function $p(z)$, where $z$ is real variable.

Differentiating (9) with respect to $z$, we obtain conditions for critical values of the parameter:

from here

$$
\frac{d p}{d z}=k z^{k-1}-\frac{n-k}{z^{n-k+1}}=0
$$

$$
\begin{aligned}
& z_{*}=\left(\frac{n-k}{k}\right)^{1} / n, \\
& p_{*}=\left(z_{*}\right)^{k}+\frac{1}{z_{*}^{n-k}} .
\end{aligned}
$$

In accordance with the general scheme of studying of (5)-(7), the locus of complex roots of equation (8) on the complex plane is defined according to (9), taking into account the condition $\operatorname{Im} p(z)=0$. 


\section{Specific examples}

Implementation of the general scheme of construction of the root locus of algebraic equation (8) is illustrated by some specific examples.

Let us start with the simplest quadratic equation

$$
z^{2}-p z+1=0 .
$$

From (13) we have the locus for real roots

$$
p=z+\frac{1}{z} .
$$

Relation (14) is shown in Fig. 1. The critical value of parameter $p_{*}$ is

$$
p_{*}=2, \quad z_{*}=1 .
$$

From the condition $\operatorname{Im} p=0$ for (14) we have: $a^{2}+b^{2}=1$ on the complex plane $(a, b)$, i.e., for various $p$ complex roots lie on the unit circle (see Fig. 2). Let us consider a cubic equation

$$
z^{3}-p z+1=0 .
$$

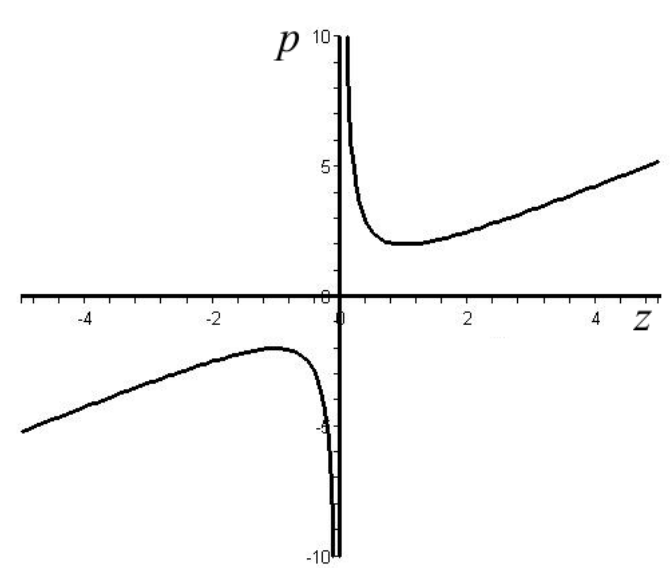

Fig. 1. Locus of real roots of equation (13)

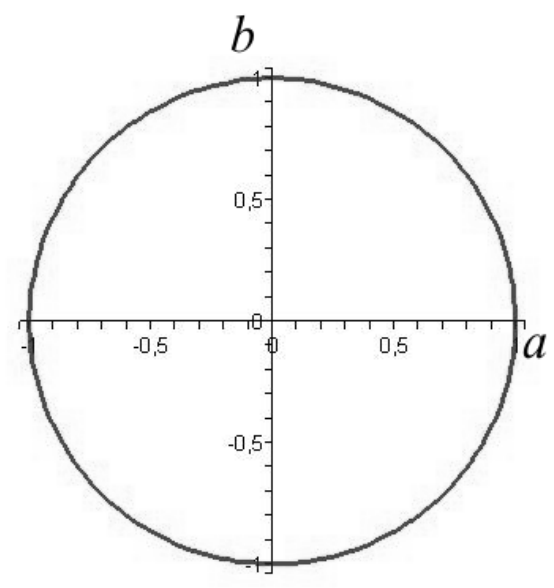

Fig. 2. Locus of complex roots $a^{2}+b^{2}=1$

It is easy to show that any cubic equation

$$
a_{3} x^{3}+a_{2} x^{2}+a_{1} x+a_{0}=0
$$

is reduced to (16), using shift and stretch of argument $x$. Equation (16) contains a single parameter $p$. From (16) we have

$$
p=z^{2}+\frac{1}{z} .
$$

Relation (17) defines the locus of real roots of cubic equation (16) (see Fig. 3). Critical values of the parameter and variable are

$$
p_{*}=\sqrt[3]{2}+\frac{1}{\sqrt[3]{4}}, \quad z_{*}=\frac{1}{\sqrt[3]{2}} .
$$

It follows from the condition $\operatorname{Im} p=0$ and (17) that locus of complex roots of equation (16) on the complex plane $(a, b)$ is

$$
a^{2}+b^{2}=\frac{1}{2 a} .
$$




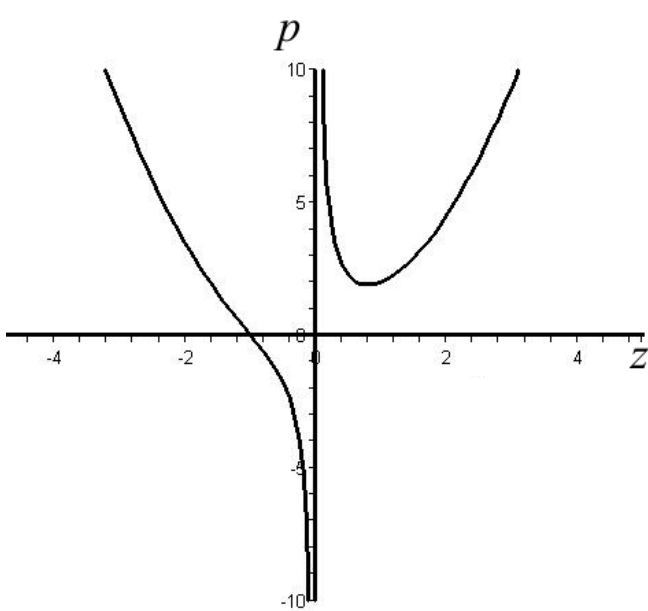

Fig. 3. Locus of real roots of equation (16)

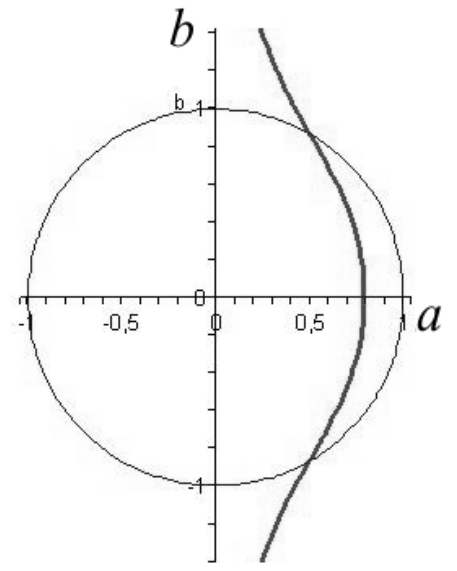

Fig. 4. Locus of complex roots $a^{2}+b^{2}=1 / 2 a$

At $b=0$ we have $a=1 / \sqrt[3]{2}$. Relation (19) is shown in Fig. 4 .

For the cubic equation

we have

$$
z^{3}-p z^{2}+1=0
$$

$$
p=z+\frac{1}{z^{2}} \text {. }
$$

The loci of real and complex roots of (20) are presented in Fig. 5 and Fig. 6. On the complex plane the roots of equation (21) lie on the curve

$$
\left(a^{2}+b^{2}\right)^{2}=2 a .
$$

The critical values $p_{*}$ and $z_{*}$ are defined in (18).

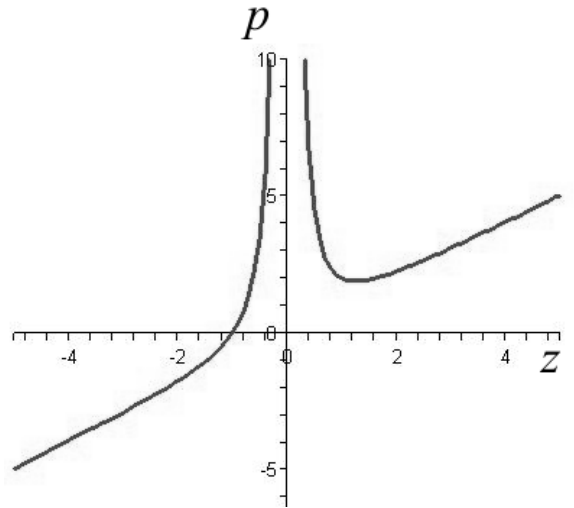

Fig. 5. Locus of real roots of equation (20)

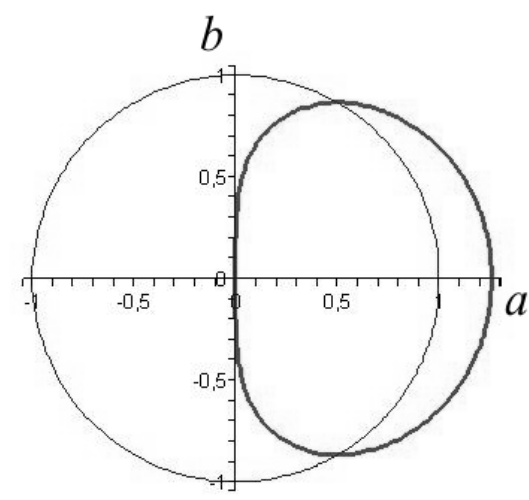

Fig. 6. Locus of complex roots (22)

Let us consider several variants of the 4th degree equation. For equation

$$
z^{4}-p z+1=0
$$

real roots are specified by relation

$$
\begin{gathered}
p=z^{3}+\frac{1}{z} . \\
-144-
\end{gathered}
$$


Complex roots are defined as above, using condition $\operatorname{Im} p=0$ :

$$
\left(3 a^{2}-b^{2}\right)\left(a^{2}+b^{2}\right)=1
$$

Relations (24) and (25) are illustrated in Fig. 7 and Fig. 8, where

$$
p_{*}= \pm\left(\sqrt[4]{3}+\frac{1}{\sqrt[3]{81}}\right), \quad z_{*}= \pm \frac{1}{\sqrt[4]{3}}
$$

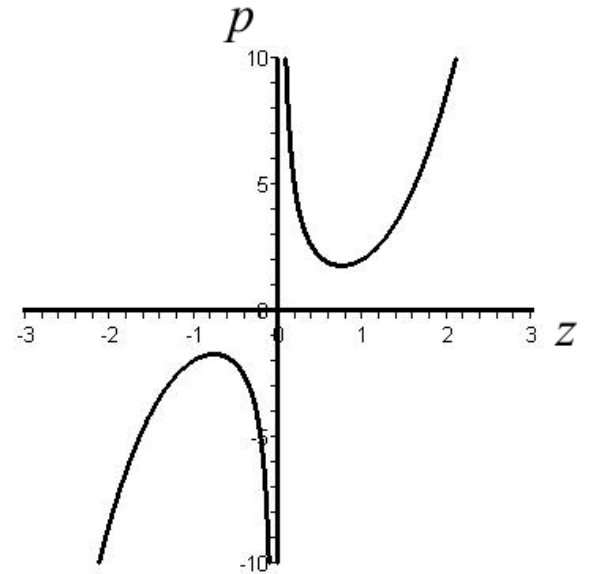

Fig. 7. Locus of real roots of equation (23)

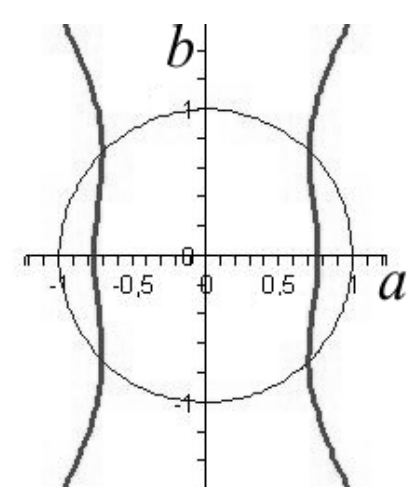

Fig. 8. Locus of complex roots (25)

For the 4th degree equation of the form

$$
z^{4}-p z^{2}+1=0
$$

we have

$$
p=z^{2}+\frac{1}{z^{2}} ; \quad \operatorname{Im} p=0:\left(a^{2}+b^{2}\right)^{3}=1 .
$$

The loci of real and complex roots are shown in Fig. 9 and Fig. 10. In this case

$$
p_{*}=2, \quad z_{*}= \pm 1
$$

For the 4th degree equation of the form

$$
z^{4}-p z^{3}+1=0
$$

we have

$$
\begin{gathered}
p=z+\frac{1}{z^{3}} \\
\operatorname{Im} p=0: \quad\left(a^{2}+b^{2}\right)^{3}=3 a^{2}-b^{2} .
\end{gathered}
$$

The loci of real and complex roots for (28) are shown in Fig. 11 and Fig. 12. Here critical values $z_{*}$ and $p_{*}$ have the form

$$
p_{*}= \pm\left(\sqrt[4]{3}+\frac{1}{\sqrt[4]{27}}\right), \quad z_{*}= \pm \sqrt[4]{3}
$$




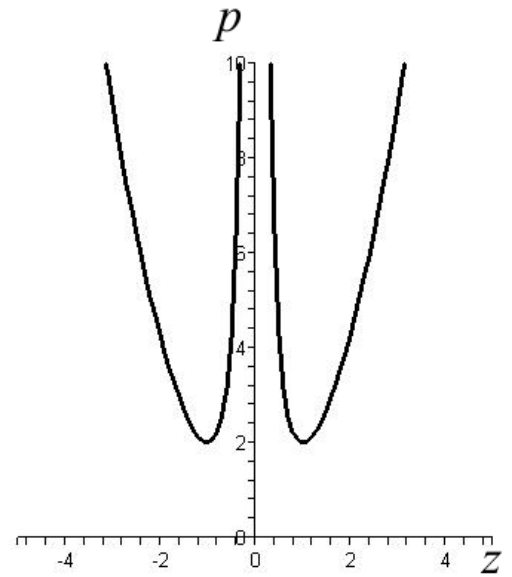

Fig. 9 I nomis of real ronts of emution (27)

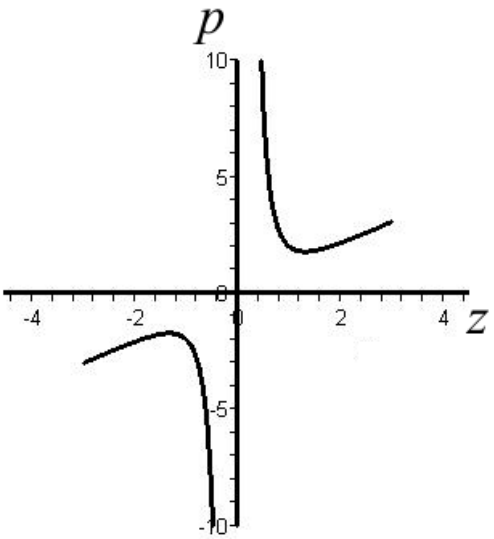

Fig. 11. Locus of real roots of equation (28)

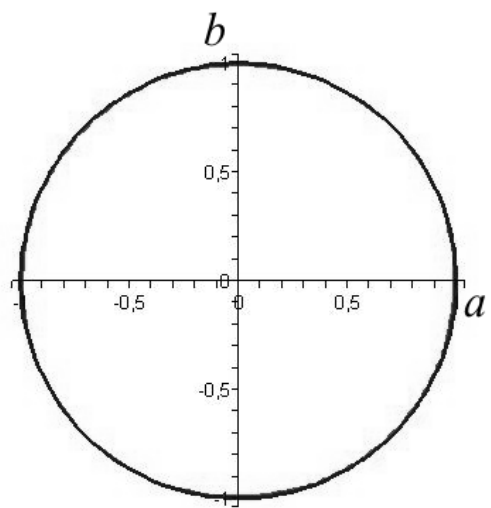

Fig. 10. Locus of complex roots $\left(a^{2}+b^{2}\right)^{2}=1$

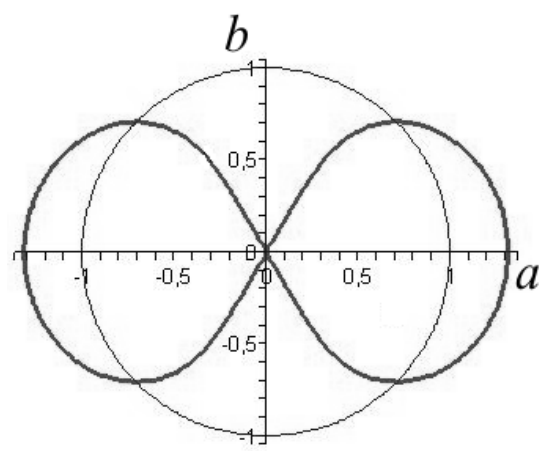

Fig. 12. Locus of complex roots (30)

Consider equations of higher degree. For the 5th degree equation

$$
z^{5}-p z+1=0
$$

we have

$$
\begin{gathered}
p=z^{4}+\frac{1}{z} \\
\operatorname{Im} p=0: \quad 4 a\left(a^{4}-b^{4}\right)=1 .
\end{gathered}
$$

The loci of real and complex roots of (32) and (33) are shown in Fig. 13 and Fig. 14. Critical values $p_{*}$ and $z_{*}$ are

$$
p_{*}=\sqrt[5]{4}+\frac{1}{z_{*}^{4}}, \quad z_{*}=\sqrt[5]{4}
$$

The loci of real and complex roots of algebraic 5 th degree equations

$$
z^{5}-p z^{3}+1=0
$$

and

$$
z^{5}-p z^{4}+1=0
$$




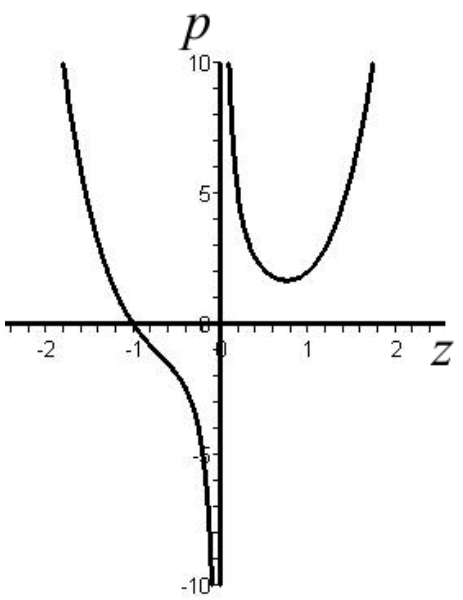

Fig. 13. Locus of real roots of equation (31)

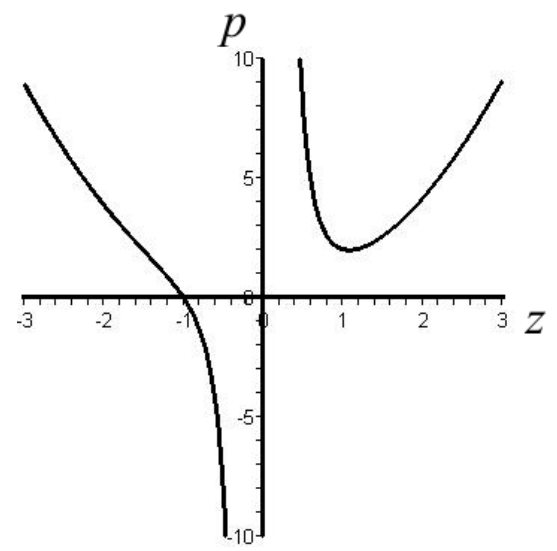

Fig. 15. Locus of real roots of equation (34)

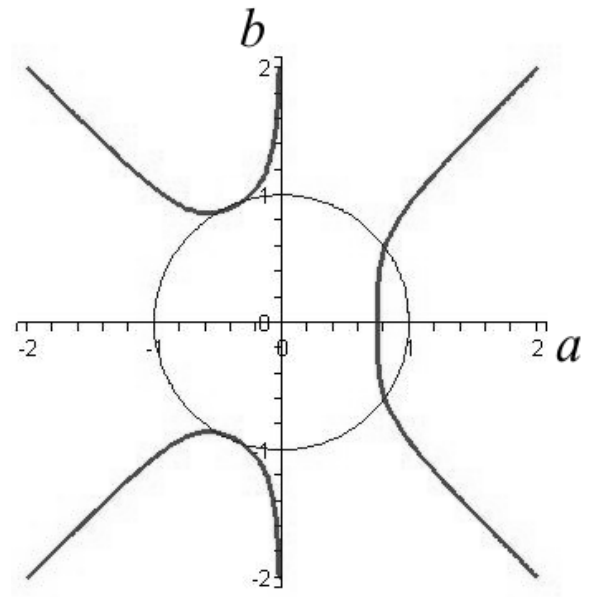

Fig. 14. Locus of complex roots (33)

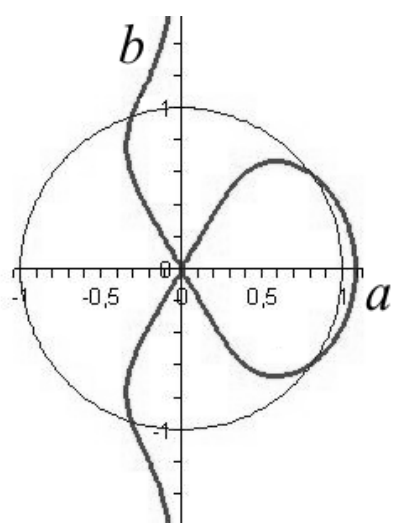

Fig. 16. Locus of complex roots of equation (34)

are presented in Figs. 15-18.

The loci of complex roots of equations of higher degree

$$
z^{8}-p z+1=0
$$

and

$$
z^{8}-p z^{7}+1=0
$$

are shown in Figs. 19, 20.

Presented results allows us to estimate by analogy the qualitative nature of the corresponding parametric portraits of various types of algebraic equations of higher degree. For example, the loci of complex roots of equations

$$
z^{9}-p z^{8}+1=0
$$

and

$$
z^{10}-p z^{9}+1=0
$$

are shown in Fig. 21 and 22. 


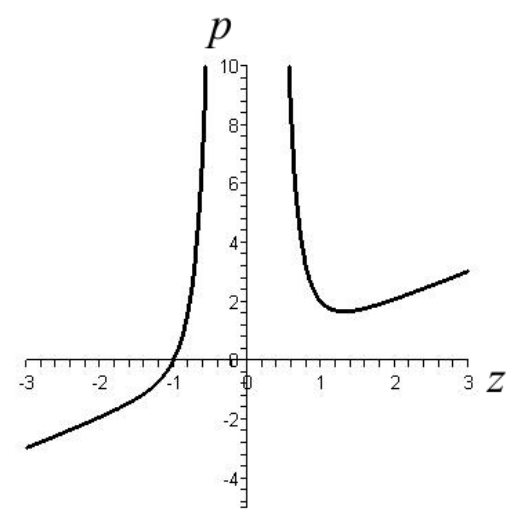

Fig. 17. Locus of real roots of equation (35)

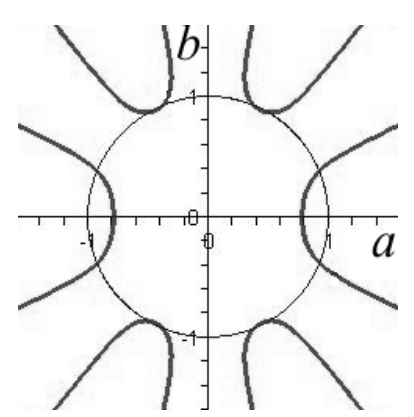

Fig. 19. Locus of real roots of equation (36)

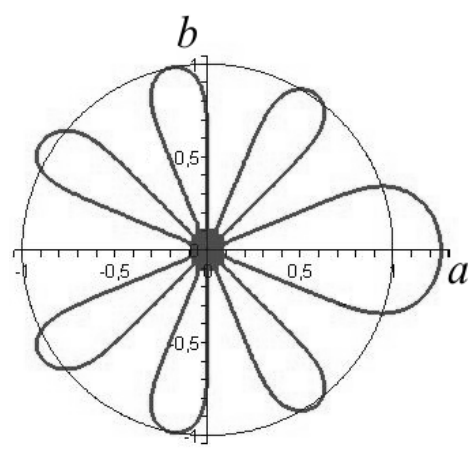

Fig. 21. Locus of complex roots of equation (38) Fig. 22. Locus of complex roots of equation (39)

\section{Conclusion}

Comparative analysis of algebraic equations (38), (39) allows us to conclude that complex roots of equations

$$
z^{m}-p z+1=0
$$

at large $m$, rather densely fill the whole complex plane except the inside of unit circle with the centre at the origin. On the contrary, for equations

$$
z^{m}-p z^{m-1}+1=0
$$


complex roots are located inside of unit circle with the centre at the origin. For equations

$$
z^{m}-p z^{m-k}+1=0
$$

where $k$ changes from 1 to $m-1$, the situation is intermediate between situations for (40) and (41). One part of the branches of the root locus on the complex plane goes to infinity, and other part is localized in a neighbourhood of the origin.

The performed analysis of several specific cases allows us to do some conclusions for algebraic equations of more general type

$$
z^{m}+a_{1} z^{m-1}+\ldots+a_{m-1} z+1=0
$$

As real parameters $a_{i}$ change, the loci of real roots of equation (43) can be easily determined by analogy with the performed analysis. To determine the locus of complex roots of equation (43) the results for some special cases presented in Figs. 9-22 will be undoubtedly useful.

Calculations show that a parametric portrait of an algebraic equation significantly depends on the type of an equation. Trinomial equations are simply analyzed. In typical case, the number of branches on which the real and complex roots are located is determined by the number of nonzero terms of this equation. The character of the localization of complex roots depends on the equation degree and degree of unknown variable at which the varying parameter is set.

Thus, the presented elementary numerical and qualitative analysis of specific algebraic equations allows us not only to get specific results of the analysis of the parameter dependence of equation roots but also to work out the understanding of qualitative features of curves on which roots of algebraic equations are located. It gives us the possibility to predict the qualitative behaviour of these roots as parameters change over a wide range.

\section{References}

[1] R.Aris, Introduction to the analysis of chemical reactors, Englewood Cliffs, NJ, USA, Prentice-Hall, 1965.

[2] V.I.Bykov, Modeling of critical phenomena in chemical kinetics, Moscow, URSS, 2014 (in Russian).

[3] V.I.Bykov, S.B.Tsybenova, Nonlinear models of chemical kinetics, Moscow, URSS, 2011 (in Russian).

[4] B.V.Volter, I.E.Sal'nikov, Stability of work regimes of the chemical reactors, Moscow, Khimiya, 1982 (in Russian).

[5] A.G. Kurosh, Course of higher algebra, Moscow, Nauka, 1965.

[6] V.I.Bykov, A.M.Kytmanov, M.Z.Lazman, Elimination methods in polynomial computer algebra, Novosibirsk, Nauka, 1991 (in Russian).

[7] V.I.Bykov, A.M.Kytmanov, M.Z.Lazman, M.Passare (ed.), Elimination methods in polynomial computer algebra, Netherlands, Springer Science and Business Media, 2012.

[8] B.P.Demidovich, Lectures on stability theory, Moscow, Nauka, 1967.

[9] F.Klein, Lectures on the icosahedron and the solution of equations of the fifth degree, NY, USA, Dover Publ., 1956. 
[10] F.Klein, Elementary Mathematics from an Advanced Standpoint: Vol. I. Arithmetic, Algebra, Analysis, NY, USA, Dover Publ., 2004.

[11] F.R.Gantmakher, The theory of matrices, Moscow, Nauka, 1967.

[12] V.I.Arnold, Catastrophe theory, Moscow, Znanie, 1981.

[13] T.Poston, I.Stewart, Catastrophe theory and its applications, NY, USA, Dover Publ., 1996.

\section{Геометрическое место корней алгебраических уравнений}

Валерий И. Быков

Светлана Б. Цыбенова

Институт биохимической физики им. Н. М. Эмануэля РАН

Москва, Российская Федерация

Аннотация. В работе построено геометрическое место действительных и комплексных корней алгебраических уравнений. Расчеты конкретных уравнений показывают, что расположение этих корней существенно зависит от вида уравнения.

Ключевые слова: алгебраические уравнения, действительные и комплексные корни, геометрическое место корней, нелинейные уравнения с параметрами. 